\title{
SINTOMAS E SINAIS DE RESPIRAÇÃO \\ PREDOMINANTEMENTE ORAL EM ADOLESCENTES COM TRANSTORNO DE DÉFICIT DE ATENÇÃO/HIPERATIVIDADE E QUEIXA DE PREJUÍZO ESCOLAR
}

\author{
Symptoms and signs of mouth breathing in teenagers \\ with attention deficit hyperactivity disorder and with complaints \\ about school underachievement
}

Tatiana Leonel da Silva Costa (1), Dioclécio Campos Júnior ${ }^{(2)}$, Hilton Justino da Silva ${ }^{(3)}$, Daniele Andrade da Cunha ${ }^{(4)}$

\section{RESUMO}

Objetivos: caracterizar sintomas e sinais da Respiração Predominantemente Oral em adolescentes com Transtorno de Déficit de Atenção/Hiperatividade e queixa de prejuízo escolar e relacionar as características com os Tipos do Transtorno de Déficit de Atenção/Hiperatividade, gênero e faixa etária. Métodos: realizada no ADOLESCENTRO, instituição pública do Distrito Federal, com 115 adolescentes de ambos os gêneros, faixa etária 10-18 anos, apresentando queixa de prejuízo escolar e Transtorno do Déficit de Atenção/Hiperatividade. Histórico dos sintomas diurnos e noturnos dos dois aos doze anos foi analisado e sinais da Respiração Predominantemente Oral. Resultados: alta ocorrência (86,95\%) de sintomas e sinais da Respiração Predominantemente Oral. Registrou-se associação entre amigdalite e tipos do Transtorno de Déficit de Atenção/Hiperatividade com maior frequência no Tipo II hiperativo (60\%), sialorreia no travesseiro com gênero predominando o masculino $(65,5 \%)$ e respiração oral diurna $(64,7 \%)$, respiração oral noturna $(82,4 \%)$ e sialorreia no travesseiro $(64,7 \%)$ todas estas com a faixa etária de 10 a 12 anos. Houve associação entre lábios $(54,3 \%)$ e língua (54,3\%) não funcionais e faixa etária de 10 a 12 anos. Encontrou-se diferença estatisticamente significante para expulsões (20\%), suspensões (70\%), advertências (100\%) e tipos de Transtorno de Déficit de Atenção/Hiperatividade com maior freqüência no Tipo II hiperativo. Conclusão: avaliação da respiração em pacientes com Transtorno do Déficit de Atenção/Hiperatividade e queixa de prejuízo escolar é fundamental, pois dificuldades escolares podem estar associadas à história pregressa de respiração oral e Distúrbios Respiratórios do Sono comuns nestes quadros.

DESCRITORES: Transtorno da Falta de Atenção com Hiperatividade; Respiração Bucal; Sono; Transtornos Respiratórios; Baixo Rendimento Escolar; Adolescente

(1) Fonoaudióloga; Professora Adjunta do Curso de Graduação em Fonoaudiologia do Centro Universitário Planalto do Distrito Federal, UNIPLAN, Brasília, DF; Mestre em Ciências da Saúde pela Universidade de Brasília.

(2) Médico; Professor Titular da Universidade de Brasília, UNB, Brasília, DF; Presidente da Sociedade Brasileira de Pediatria; Doutor em Pediatria pela Universiti Libre de Bruxelles, Bélgica.

(3) Fonoaudiólogo; Professor Adjunto do Curso de Graduação em Fonoaudiologia da Universidade Federal de Pernambuco, UFPE, Recife, PE; Doutor em Nutrição pela Universidade Federal de Pernambuco.

(4) Fonoaudióloga; Coordenadora do Curso de Graduação em Fonoaudiologia da Faculdade Integrada do Recife, FIR, Recife, PE; Mestre em Nutrição pela Universidade Federal de Pernambuco.

Conflito de interesses: inexistente

\section{INTRODUÇÃO}

Nos últimos anos, a dificuldade escolar deixou de ser foco apenas da área da educação e passou a ser queixa frequente nos ambulatórios e consultórios de profissionais da área de saúde. Concomitantemente a investigações sobre acuidade visual e auditiva, as alterações comportamentais na fase escolar passaram a ter atenção especial na área da saúde, recebendo um foco diferente do que era preconizado até então como o reflexo de má educação, desmotivação, negligência ou "preguiça". 
O Transtorno de Déficit de Atenção/Hiperatividade (TDAH) é um dos diagnósticos mais comuns na fase escolar. É caracterizado pela tríade desatenção, hiperatividade e impulsividade, comportamentos que interferem negativamente na vida escolar ${ }^{1,2}$.

As crianças que apresentam o diagnóstico do TDAH, mesmo com o potencial intelectual adequado, estão fadadas ao fracasso. Os erros na escola podem ser justificados por comportamentos desencadeados pelos sintomas presentes neste transtorno ${ }^{3}$.

Estas crianças cometem erros por desatenção ou por não conseguirem estudar o suficiente, uma vez que não ficam muito tempo sentadas. $O$ baixo rendimento escolar acontece como consequência e pode propiciar a baixa auto-estima e desencadear uma complexa desordem comportamental, levando ao comprometimento da vida escolar, social, emocional e familiar ${ }^{1,3}$.

$\mathrm{Na}$ adolescência e na vida adulta este quadro persiste de 70 a $85 \%$ dos casos. Nestas fases a desatenção e a impulsividade prevalecem, mas continuam acompanhadas dos prejuízos, o que inclui os reflexos na vida acadêmica ${ }^{4}$.

Os sintomas do TDAH, mencionados por vários autores, também podem ser encontrados nas pessoas que respiram pela boca. Agitação, impaciência, ansiedade, impulsividade, desânimo, dificuldade de atenção, concentração e irritação são características comportamentais de indivíduos com Respiração Predominantemente Oral (RPO), as quais também podem propiciar as dificuldades escolares ${ }^{5}$.

Nos dois quadros, TDAH e na RPO, um sono não reparador, decorrente de possíveis Distúrbios Respiratórios do Sono (DRS), pode desencadear estas alterações que, juntamente com a sonolência diurna irão interferir diretamente na atenção e concentração durante as aulas e no rendimento escolar ${ }^{5,6}$.

Autores sugerem a relação entre TDAH e dificuldades escolares ${ }^{1,7,8}$. Da mesma forma, a RPO e sua relação com as dificuldades escolares já foram bastante estudadas ${ }^{9-11}$. Apesar do quadro similar em relação aos sintomas comportamentais e os decorrentes prejuízos escolares, o TDAH e a RPO são abordados de modo desvinculado na prática clínica. Raras, também, são as pesquisas que abordam estes dois assuntos em um mesmo estudo e, embora as existentes sugiram uma possível relação, há uma carência de estudos com amostra de adolescentes.

Tendo em vista o exposto, esta pesquisa teve como objetivo geral caracterizar os sintomas e sinais da RPO em adolescentes com TDAH e queixa de prejuízo escolar, e como objetivos específicos relacionar os achados sobre os sintomas e sinais da RPO com o tipo do diagnóstico do TDAH, gênero e faixa etária dos adolescentes.

\section{MÉTODOS}

Por meio de uma amostra de conveniência, foi realizado um estudo analítico observacional do tipo transversal.

A coleta dos dados foi realizada de abril a novembro de 2007, no Centro de Referência, Pesquisa, Capacitação e Atenção ao Adolescente em Família - ADOLESCENTRO, pertencente à Secretaria de Saúde do Distrito Federal.

A população foi composta por 115 adolescentes de ambos os gêneros, faixa etária de 10 a 18 anos, com queixa de prejuízo escolar e diagnóstico de TDAH realizado pela equipe multidisciplinar, baseada nos critérios do DSM-IV-1994.

Realizou-se levantamento através de programa de gestão de dados, utilizado há oito anos e denominado Sistema de Informações de Atendimento do ADOLESCENTRO-SISA. Através deste programa foram analisados os dados dos adolescentes registrados até Abril de 2007, os quais apresentavam diagnóstico de TDAH e a queixa de prejuízo escolar. Em seguida foram aplicados os critérios de inclusão e exclusão obtendo-se o número final da amostra.

Como critérios de inclusão foram estabelecidos: adolescentes de ambos os gêneros, com idades entre 10-18 anos, portadores de TDAH e que tivessem chegado aos ADOLESCENTRO coma a queixa de prejuízo escolar.

Foram estabelecidos os seguintes critérios de exclusão: comprometimento visual, auditivo, mental, depressão e vivência de violência sexual, os quais pudessem afetar diretamente o desempenho escolar. Excluíram-se da amostra também, os adolescentes que apresentavam obesidade, pois este quadro pode justificar per se aspectos do sono investigados. Foi possível aplicar os critérios de exclusão, tendo em vista que todos os respectivos diagnósticos já haviam sido realizados pela equipe médica e encontravam-se registrados em cada prontuário.

Em um primeiro momento, o adolescente e o responsável foram convidados a participarem da pesquisa, sendo informados do objetivo e da forma de coleta dos dados. Em seguida o Termo de Consentimento Livre e Esclarecido foi lido, assinado pelo responsável, os dados foram colhidos, o que totalizou o tempo máximo de 10 minutos por paciente.

Variáveis do adolescente: idade, gênero, diagnóstico do tipo de TDAH e escolaridade. A queixa 
de prejuízo escolar referente à repetência e expulsões em toda vida escolar e recuperação, advertências e suspensões no ano anterior a entrada do adolescente no ADOLESCENTRO.

O histórico dos sintomas diurnos e noturnos dos dois aos doze anos foi analisado assim como alguns sinais relatados pela literatura, como características comuns da RPO. Os sintomas investigados foram: respiração oral diurna, resfriados constantes, rinite, amigdalite de repetição, respiração oral noturna, ronco, sialorreia no travesseiro e pausas respiratórias. No que se refere aos sinais as variáveis pesquisadas foram olheiras, halitose, salivação excessiva, lábios ressecados, lábios funcionais (ocluídos), não funcionais (abertos ou entreabertos), língua funcional (por trás dos incisivos superiores) e não funcional (anteriorizada, ou no assoalho bucal).

Na Avaliação da Função Nasal utilizou-se o teste do Espelho Nasal Milimetrado de Altmann conforme instruções da embalagem. A condensação referente às duas narinas foi registrada como positiva, e negativa a referente a apenas uma narina.

Todos os adolescentes que apresentaram sintomas e sinais da $\mathrm{RO}$ receberam orientações e foi explanada a necessidade de um possível tratamento. Em seguida, foram registrados no prontuário os dados obtidos de cada paciente e estes encaminhados para avaliação otorrinolaringológica, realizada em hospitais da rede da Secretaria de Estado de Saúde do Distrito Federal.

O projeto desta pesquisa foi submetido ao Comitê de Ética em Pesquisa da Secretaria de Saúde do Distrito Federal, sendo aprovado sem restrições através do Protocolo №. 037/07.

Para a análise estatística foi utilizado software estatístico $S A{ }^{\circledR}{ }^{\circledR}$ versão 8.2. Utilizando-se estatística descritiva, foi apontada através de frequência absoluta e relativa a prevalência do diagnóstico dos tipos de TDAH e os sintomas e sinais de RPO. O teste Qui-quadrado foi utilizado para verificar possíveis diferenças entre as variáveis estudadas nesta pesquisa. Para aceitação da associação admitiu-se nível de confiança de $5 \%(p=0,05)$.

\section{RESULTADOS}

A amostra desta pesquisa foi constituída por 115 adolescentes.

No diagnóstico do Tipo do TDAH, o Tipo III combinado foi o mais frequente sendo encontrado em 71 adolescentes $(61,74 \%)$, seguido pelo Tipo I predominantemente desatento encontrado em 34 adolescentes $(29,57 \%)$. Já o TIPO II predominantemente hiperativo foi constatado em 10 sujeitos (8,7\%) (Tabela 1).

Houve diferença estatisticamente significante em relação aos tipos de TDAH quanto ao gênero, com maior ocorrência no masculino $(p=0,002)$. No gênero masculino, o tipo de TDAH mais encontrado foi o TIPO II sendo observado em 9 meninos (90\%) e no gênero feminino o TIPO I observado em 16 meninas $(47,6 \%)$. Para a faixa etária e o grau de escolaridade não foi encontrada diferença significante na amostra (Tabela 2).

Da amostra, 86,95\% apresentaram sintoma ou sinal de RPO. Apenas 39 adolescentes (33,91\%) realizaram algum tratamento. Destes 33 sujeitos $(28,70 \%)$ foram submetidos a tratamentos medicamentosos (Tabela 3).

Os sintomas mais frequentes, encontrados em mais da metade da amostra foram o ronco mencionado por 76 adolescentes $(66,09 \%)$, a respiração oral noturna por $75(65,22 \%)$, a rinite alérgica por $73(63,48 \%)$ e sialorreia no travesseiro por 65 $(56,52 \%)$. A pausa respiratória durante o sono foi o sintoma menos relatado pelos informantes sendo registrada em 38 adolescentes (33,04\%) (Tabela 3).

Os sinais da RPO referentes a olheiras encontrado em 68 adolescentes $(59,13 \%)$ e lábios ressecados em 60 adolescentes $(52,17 \%)$ foram observados em pouco mais da metade da população. Houve baixa ocorrência de salivação excessiva observada em 47 sujeitos (40,87\%), halitose em 41 sujeitos $(35,65 \%)$ e postura inadequada de lábios e língua, sendo registrados 45 sujeitos apresentando $(39,13 \%)$ lábios não funcionais e 43 (37,39\%) língua não funcional (Tabela 4).

A respeito da medição da aeração nasal, realizada por meio do registro no Espelho Milimetrado de Altmann, 93 adolescentes (80,87\%) apresentaram saída de ar bilateral, ou seja, condensação

Tabela 1 - Distribuição da amostra em números absolutos e percentuais do tipo do diagnóstico do transtorno de déficit

\begin{tabular}{lcc}
\hline TIPO Diagnóstico TDAH & N & $\%$ \\
\hline Tipo I - predominantemente desatento & 34 & 29,57 \\
Tipo II - predominantemente hiperativo & 10 & 8,70 \\
Tipo III - combinado & 71 & 61,74 \\
\hline
\end{tabular}


Tabela 2 - Distribuição da amostra segundo faixa etária, gênero, escolaridade e tipo de escola em relação aos tipos do transtorno de déficit de atenção/hiperatividade

\begin{tabular}{|c|c|c|c|c|c|c|c|c|c|}
\hline & \multicolumn{2}{|c|}{ TIPO I } & \multicolumn{2}{|r|}{ TIPO II } & \multicolumn{2}{|c|}{ TIPO III } & \multicolumn{2}{|c|}{ TOTAL } & \multirow[t]{2}{*}{$\mathrm{P}^{*}$} \\
\hline \multicolumn{9}{|l|}{ Faixa etária } & \\
\hline 10 a 12 & 14 & $41,18 \%$ & 3 & $30,00 \%$ & 18 & $25,35 \%$ & 35 & $30,43 \%$ & \multirow{3}{*}{0,4303} \\
\hline 13 a 15 & 15 & $44,12 \%$ & 4 & $40,00 \%$ & 40 & $56,34 \%$ & 59 & $51,30 \%$ & \\
\hline 16 a 18 & 5 & $14,71 \%$ & 3 & $30,00 \%$ & 13 & $18,31 \%$ & 21 & $18,26 \%$ & \\
\hline \multicolumn{10}{|l|}{ Gênero } \\
\hline Masculino & 18 & $52,94 \%$ & 9 & $90,00 \%$ & 59 & $83,10 \%$ & 86 & $74,78 \%$ & \multirow{2}{*}{0,0020} \\
\hline Feminino & 16 & $47,06 \%$ & 1 & $10,00 \%$ & 12 & $16,90 \%$ & 29 & $25,22 \%$ & \\
\hline \multicolumn{10}{|l|}{ Escolaridade } \\
\hline $1^{\circ} \mathrm{Grau}$ & 29 & $85,29 \%$ & 10 & $100,00 \%$ & 67 & $94,37 \%$ & 106 & $92,17 \%$ & \multirow{2}{*}{0,1692} \\
\hline $2^{\circ} \mathrm{Grau}$ & 5 & $14,71 \%$ & 0 & $0,00 \%$ & 4 & $5,63 \%$ & 9 & $7,83 \%$ & \\
\hline \multicolumn{10}{|l|}{ Tipo de Escola } \\
\hline Pública & 20 & $58,82 \%$ & 10 & $100,00 \%$ & 55 & $77,46 \%$ & 85 & $73,91 \%$ & \multirow{2}{*}{0,0182} \\
\hline Particular & 14 & $41,18 \%$ & 0 & $0,00 \%$ & 16 & $22,54 \%$ & 30 & $26,09 \%$ & \\
\hline
\end{tabular}

* Teste Qui-quadrado - Valor $\mathrm{p}=0,05$

Tabela 3 - Distribuição da amostra em números absolutos e percentuais segundo a caracterização dos sintomas da respiração predominantemente oral dos 2 aos 12 anos

\begin{tabular}{|c|c|c|c|}
\hline Sintomas & & $\mathbf{N}$ & $\%$ \\
\hline Resfriados constantes & & 47 & 40,87 \\
\hline Rinite & & 73 & 63,48 \\
\hline Amidalite* & & 41 & 35,65 \\
\hline Respiração oral diurna* & & 57 & 49,57 \\
\hline Respiração oral noturna* & & 75 & 65,22 \\
\hline Ronco & & 76 & 66,09 \\
\hline Sialorreia* & & 65 & 56,52 \\
\hline Pausas respiratórias* & & 38 & 33,04 \\
\hline \multirow[t]{5}{*}{ Realizou tratamento* } & & 39 & 33,91 \\
\hline & Qual tratamento & & \\
\hline & Medicamentoso & 33 & $28,70 \%$ \\
\hline & Cirúrgico & 3 & $2,61 \%$ \\
\hline & Ambos & 3 & $2,61 \%$ \\
\hline
\end{tabular}

* Nota: Alguns dados sem informação

Tabela 4 - Distribuição da amostra em números absolutos e percentuais segundo a caracterização dos sinais da respiração predominantemente oral

\begin{tabular}{|c|c|c|c|}
\hline Sinais & & $\mathbf{N}$ & $\%$ \\
\hline Olheiras & & 68 & 59,13 \\
\hline Halitose & & 41 & 35,65 \\
\hline Salivação excessiva & & 47 & 40,87 \\
\hline Lábios ressecados & & 60 & 52,17 \\
\hline \multicolumn{4}{|l|}{ Postura de lábios } \\
\hline & Não funcionais & 45 & 39,13 \\
\hline \multicolumn{4}{|l|}{ Postura de língua } \\
\hline & Não funcional & 43 & 37,39 \\
\hline
\end{tabular}


no espelho referente às duas narinas. Destes, 49 $(52,68 \%)$ apresentaram simetria na saída de ar bilateral registrada no espelho (Tabela 5).

Ao se relacionar os sintomas da RPO com os tipos de TDAH, foi encontrada diferença estatisticamente significante em relação aos portadores de amigdalite e repetição $(p=0,0037)$. Não houve diferença significante em relação aos demais sintomas. (Tabela 6)

Também não foi encontrada diferença estatística entre os tipos de TDAH quanto aos sinais da RPO.

$\mathrm{Na}$ análise da relação do gênero com os sintomas da RPO, foi encontrada diferença estatisticamente significante para os portadores de sialorreia durante o sono $(p=0,0036)$. A diferença estatística não pôde ser confirmada em relação às demais variáveis, consideradas as diferenças de gênero (Tabela 7).

Não houve diferença significante entre os sinais de RPO quando referidos à diferença de gênero.

Ao se relacionar faixas etárias com sintomas da RPO, foi encontrada diferença estatisticamente significante no que se refere à respiração oral diurna $(p=0,0331)$, respiração oral noturna $(p=0,0360)$ e sialorreia durante o sono $(\mathrm{p}=0,0420)$. A faixa etária que houve maior incidência destes sintomas foi dos 10 aos 12 anos, contudo respiração oral noturna foi encontrada em 28 adolescentes (82,4\%) sobressaindo-se em relação à respiração oral diurna em 22 adolescentes $(64,7 \%)$ e sialorreia durante o sono encontrada em 23 (65,7\%). Nas demais variáveis, a diferença não foi significante (Tabela 8).

A diferença estatisticamente significante foi observada em relação a lábios não funcionais $(p=0,0300)$ e à língua não funcional $(p=0,0224)$, ambas variáveis sendo encontradas com maior frequência na faixa etária dos 10 aos 12 anos. Não houve diferença estatística para as variáveis olheiras, halitose, salivação excessiva e lábios ressecados, quando avaliadas em função da faixa etária dos sujeitos (Tabela 9).

Relacionando os tipos de TDAH com as queixas de prejuízo escolar, foi encontrada diferença estatisticamente significante em relação a expulsões $(p=0,0242)$, suspensões $(p<0,0001)$ e advertências $(p<0,0001)$, havendo maior frequência destas variáveis no Tipo II - predominantemente hiperativo (Tabela 10).

Tabela 5 - Distribuição da amostra em números absolutos e percentuais segundo a medida de aeração nasal

\begin{tabular}{lrcc}
\hline Teste do Espelho de Altmann & $\mathbf{N}$ & $\%$ \\
\hline Saída de ar bilateral & Sim & 93 & 80,87 \\
& Não & 22 & 19,13 \\
Nos casos de respostas positivas & & \\
& Saída de ar maior à & & \\
& Direita & 19 & 20,43 \\
& Esquerda & 25 & 26,88 \\
& Simétrica & 49 & 52,68 \\
\hline
\end{tabular}

Tabela 6 - Distribuição da amostra segundo os tipos de transtorno de déficit de atenção/hiperatividade com relação aos sintomas da respiração predominantemente oral

\begin{tabular}{|c|c|c|c|c|c|c|c|}
\hline \multirow{2}{*}{ SINTOMAS } & \multicolumn{2}{|c|}{ TIPO I } & \multicolumn{2}{|c|}{ TIPO II } & \multicolumn{2}{|c|}{ TIPO III } & \multirow{2}{*}{$\mathbf{P}^{*}$} \\
\hline & $\mathbf{N}$ & $\%$ & $\mathbf{N}$ & $\%$ & $\mathbf{N}$ & $\%$ & \\
\hline Respiração oral diurna** & 18 & 52,9 & 7 & 70 & 32 & 45,7 & 0,3276 \\
\hline Resfriados constantes & 15 & 44,1 & 7 & 70 & 25 & 35,2 & 0,1002 \\
\hline Rinite & 22 & 64,7 & 8 & 80 & 43 & 60,6 & 0,4820 \\
\hline Amigdalite** & 18 & 54,5 & 6 & 60 & 17 & 24,6 & 0,0037 \\
\hline Respiração oral noturna ${ }^{\star \star}$ & 23 & 67,6 & 9 & 90 & 43 & 62,3 & 0,2194 \\
\hline Ronco & 24 & 70,6 & 9 & 90 & 43 & 60,6 & 0,1477 \\
\hline Sialorreia** & 21 & 61,8 & 6 & 66,7 & 38 & 54,3 & 0,6510 \\
\hline Pausas respiratórias** & 15 & 46,9 & 5 & 50 & 18 & 27,27 & 0,0958 \\
\hline
\end{tabular}

** Nota: alguns dados sem informações

* Teste Qui-quadrado - Valor $\mathrm{p}=0,05$ 
Tabela 7 - Distribuição dos sintomas de respiração predominantemente oral de acordo com o gênero

\begin{tabular}{|c|c|c|c|c|c|}
\hline \multirow{2}{*}{ SINTOMAS } & \multicolumn{2}{|c|}{ MASCULINO } & \multicolumn{2}{|c|}{ FEMININO } & \multirow{2}{*}{$\mathbf{P}^{*}$} \\
\hline & $\mathbf{N}$ & $\%$ & $\mathbf{N}$ & $\%$ & \\
\hline Respiração oral diurna** & 45 & 52,9 & 12 & 41,4 & 0,2823 \\
\hline Resfriados constantes & 39 & 45,3 & 8 & 27,6 & 0,0924 \\
\hline Rinite & 55 & 64,0 & 18 & 62,1 & 0,8554 \\
\hline Amidalite $^{\star \star}$ & 30 & 36,1 & 11 & 37,9 & 0,8635 \\
\hline Respiração oral noturna** & 58 & 69,0 & 17 & 58,6 & 0,3055 \\
\hline Ronco & 57 & 66,3 & 19 & 65,5 & 0,9403 \\
\hline Sialorreia** & 55 & 65,5 & 10 & 34,5 & 0,0036 \\
\hline Pausas respiratórias ${ }^{\star *}$ & 29 & 36,2 & 9 & 32,1 & 0,6953 \\
\hline
\end{tabular}

** Nota: Alguns dados sem informação

* Teste Qui-quadrado - Valor $\mathrm{p}=0,05$

Tabela 8 - Distribuição dos sintomas de respiração predominantemente oral de acordo com as faixas etárias

\begin{tabular}{|c|c|c|c|c|c|c|c|}
\hline \multirow{2}{*}{ SINTOMAS } & \multicolumn{2}{|c|}{10 a 12} & \multicolumn{2}{|c|}{13 a 15} & \multicolumn{2}{|c|}{16 a 18} & \multirow{2}{*}{$\mathbf{P}^{*}$} \\
\hline & $\mathbf{N}$ & $\%$ & $\mathbf{N}$ & $\%$ & $\mathbf{N}$ & $\%$ & \\
\hline Respiração oral diurna** & 22 & 64,7 & 29 & 49,2 & 6 & 28,6 & 0,0331 \\
\hline Resfriados constantes & 17 & 48,6 & 22 & 37,3 & 8 & 38,1 & 0,5382 \\
\hline Rinite & 24 & 68,6 & 39 & 66,1 & 10 & 47,6 & 0,2411 \\
\hline Amidalite $^{\star \star}$ & 16 & 48,5 & 17 & 28,8 & 8 & 40,0 & 0,1612 \\
\hline Respiração oral noturna** & 28 & 82,4 & 37 & 62,7 & 10 & 50,0 & 0,0360 \\
\hline Ronco & 27 & 77,1 & 37 & 62,7 & 12 & 57,1 & 0,2278 \\
\hline Sialorreia** & 23 & 65,7 & 35 & 61,4 & 7 & 33,3 & 0,0420 \\
\hline Pausas respiratórias ${ }^{\star \star}$ & 15 & 46,9 & 18 & 32,1 & 5 & 25,0 & 0,2171 \\
\hline
\end{tabular}

** Nota: Alguns dados sem informação

* Teste Qui-quadrado - Valor $\mathrm{p}=0,05$

Tabela 9 - Distribuição dos sinais de respiração predominantemente oral de acordo com as faixas etárias

\begin{tabular}{lccccccc}
\hline \multirow{2}{*}{ SINAIS } & \multicolumn{2}{c}{$\mathbf{1 0} \mathbf{a} \mathbf{1 2}$} & \multicolumn{2}{c}{$\mathbf{1 3} \mathbf{a} \mathbf{1 5}$} & \multicolumn{2}{c}{$\mathbf{1 6} \mathbf{a} \mathbf{1 8}$} & \multirow{2}{*}{$\mathbf{P}^{*}$} \\
\cline { 2 - 7 } & $\mathbf{N}$ & $\mathbf{0}$ & $\mathbf{N}$ & $\mathbf{0}$ & $\mathbf{N}$ & $\%$ & \\
\hline Olheiras & 23 & 65,7 & 33 & 55,9 & 12 & 57,1 & 0,6339 \\
Halitose & 15 & 42,9 & 21 & 35,6 & 5 & 23,8 & 0,3542 \\
Salivação excessiva & 16 & 45,7 & 22 & 37,3 & 9 & 42,9 & 0,7091 \\
Lábios ressecados & 22 & 62,9 & 28 & 47,5 & 10 & 47,6 & 0,3164 \\
Lábios não funcionais & 19 & 54,3 & 22 & 37,3 & 4 & 19,0 & 0,0300 \\
Língua não funcional & 19 & 54,3 & 20 & 33,9 & 4 & 19,0 & 0,0224 \\
\hline
\end{tabular}

* Teste Qui-quadrado - Valor $p=0,05$ 
Tabela 10 - Distribuição da amostra segundo os tipos de transtorno de déficit de atenção/ hiperatividade com relação aos prejuízos escolares

\begin{tabular}{lcccccrr}
\hline \multirow{2}{*}{ PREJUízos } & \multicolumn{2}{c}{ TIPO I } & \multicolumn{2}{c}{ TIPO II } & \multicolumn{2}{c}{ TIPO III } & \multirow{2}{*}{ P $^{*}$} \\
\cline { 2 - 6 } & $\mathbf{N}$ & $\%$ & $\mathbf{N}$ & $\%$ & $\mathbf{N}$ & $\%$ & \\
\hline Reprovação & 23 & 67,6 & 7 & 70,0 & 52 & 73,2 & 0,8351 \\
Expulsão & 0 & 0,0 & 2 & 20,0 & 3 & 4,2 & 0,0242 \\
Advertência & 9 & 26,5 & 10 & 100,0 & 42 & 59,2 & $<0,0001$ \\
Suspensão & 2 & 5,9 & 7 & 70,0 & 17 & 23,9 & $<0,0001$ \\
Recuperação & 28 & 82,4 & 9 & 90,0 & 65 & 91,6 & 0,3757 \\
\hline
\end{tabular}

* Teste Qui-quadrado - Valor p= 0,05 de atenção/hiperatividade

\section{DISCUSSÃO}

Os resultados encontrados, referentes à identificação do adolescente, estão de acordo com a literatura que, em vários estudos, registra a prevalência de TDAH no gênero masculino ${ }^{1,3,12,13}$, numa proporção de $3: 1^{13,14}$ e com predomínio do tipo combinado ${ }^{15}$ em adolescentes ${ }^{12}$.

O predomínio do gênero masculino nos portadores de TDAH talvez decorra do subdiagnóstico no gênero feminino, uma vez que meninas apresentam menos sintomas de conduta se comparadas aos meninos, propiciando uma diminuição na procura do tratamento por parte dos familiares e educadores, já que não se sentem preocupados por não perceberem o quadro ${ }^{13,14}$, o que pode justificar o resultado neste estudo.

Em relação aos tipos de TDAH, os resultados estão em consonância com a literatura, pois embora o TDAH predomine numa faixa essencialmente infantil, pode persistir em $70 \%$ a $85 \%$ dos casos na adolescência, sendo encontrado com mais frequência nesta faixa etária o tipo combinado, seguido pelo desatento 4 .

A baixa frequência do TIPO II era esperada, pois o predomínio dos sintomas de hiperatividade acomete frequentemente a faixa etária de pré-escolares, que não foi pesquisada neste estudo ${ }^{4}$.

As doenças respiratórias frequentemente instalam-se na infância, apresentando etiologias distintas e graus variados de comprometimento. Contudo, confluem na maioria dos casos para a mesma consequência: a respiração oral ${ }^{9,10,16}$. Devido a isso, foi fundamental neste estudo o rastreamento de sintomas relacionados às causas que pudessem alterar o modo respiratório dos adolescentes dos dois aos doze anos.

A rinite foi observada em um percentual elevado nos sujeitos deste estudo ${ }^{16,17}$. Estima-se que 15 a $20 \%$ da população Brasileira sofram de rinite alérgica ${ }^{17}$. No Distrito Federal, uma pesquisa realizada com adolescentes mostrou que houve um aumento significante da rinite alérgica diagnosticada em 2006 (20\%) em relação a $1996(12,7 \%)^{18}$.

Estes resultados, juntamente com os dados da presente pesquisa, apontam para a necessidade de uma maior atenção em relação à rinite nos adolescentes do Distrito Federal, visto que esta patologia é uma das doenças crônicas mais prevalentes na faixa etária escolar, interferindo diretamente na qualidade de vida.

Merece ser ressaltado ainda que, apesar de menos da metade da amostra ter referido resfriados constantes dos dois aos doze anos, os principais sintomas da rinite são: espirros em "salvas", prurido nasal, coriza hialina e obstrução nasal, os quais são frequentemente confundidos com resfriados ${ }^{18}$, o que também sugerindo melhor avaliação deste tipo de queixa.

Observou-se que pouco menos da metade da amostra referiu respiração oral diurna, enquanto que, uma elevada taxa de ocorrência de sintomas durante o sono, como a respiração oral noturna, ronco e sialorreia, foi verificada. Estes achados não são surpreendentes, visto que o sono no indivíduo que respira pela boca no geral é bastante conturbado ${ }^{5,9,19}$.

Em relação aos sinais pesquisados, foram encontrados olheiras e lábios ressecados ${ }^{20}$, salivação excessiva ${ }^{5,11}$ e halitose ${ }^{5,21}$, em ordem crescente, sendo estes sinais comumente relacionados à RPO ${ }^{11}$

Lábios não funcionais (entreabertos ou abertos) foram encontrados em menos da metade da amostra. Apesar de serem sinais comuns na RPO, lábios entreabertos não significam boca aberta. Isso porque a respiração nasal pode ser obtida não apenas pelo vedamento labial, mas também pelo contato da língua com a porção anterior ou posterior do palato duro ${ }^{5}$.

Desta forma, julgou-se importante, também a avaliação da posição habitual da língua de "língua não funcional" quando anteriorizada ou em assoalho bucal ${ }^{20,22}$, sendo encontrada em menos da metade 
da amostra. Embora estas alterações tenham sido pouco frequentes, não devem ser menosprezadas, pois a ausência de vedamento labial, juntamente com o mau posicionamento da língua, pode propiciar alterações no modo respiratório e também no Sistema Estomatognático ${ }^{23}$.

Isto é possível devido ao processo alveolar encontrar-se implantado entre a língua, os lábios e as bochechas. A separação dos lábios, juntamente com o rebaixamento da língua, impede que haja as funções de contenção e modelação dos arcos dentários, resultando na diminuição do espaço buco-naso-farígeo, causando a RPO ou intensificando-a ${ }^{23}$.

Neste estudo, utilizou-se o teste do espelho, considerado um dos mais antigos e conhecidos métodos para medição da aeração nasal. Nos resultados a maioria dos adolescentes apresentou saída de ar bilateral simétrica.

A literatura mostra que o registro no espelho é referente à expiração, todavia o paciente com obstrução das vias aéreas superiores tem dificuldade na inspiração sendo esta um crítica a este tipo de método. Talvez este aspecto tenha influenciado nos resultados, o que deve ser considerado ${ }^{10,24}$.

Apesar da crítica, o uso do espelho na medição da aeração nasal vem sendo estudado e resultados importantes têm sido divulgados. Em um estudo recente, alguns pesquisadores utilizaram o EspeIho Milimetrado de Altmann antes e após a manobra de massagem e limpeza nasal em 20 crianças, havendo um aumento significante da saída de ar, observado na condensação do espelho, ao serem comparadas as duas medições ${ }^{24}$.

No que se refere ao predomínio de uma das narinas ou a assimetria na saída de ar, os resultados em percentuais do nosso estudo parecem não relevantes. Diferentemente do estudo mencionado anteriormente foi realizada apenas uma mensuração nessa amostra. Apesar disso, esses resultados em relação à assimetria na saída de ar concordam com os achados deste estudo quando os autores descrevem como não havendo um predomínio da obstrução quanto à lateralidade da narina ${ }^{24}$.

$\mathrm{Na}$ análise da relação entre os sintomas da RPO e os Tipos do TDAH, a diferença estatisticamente significante foi encontrada em relação à amigdalite de repetição. Apesar de não ser possível afirmar se os adolescentes que apresentaram respostas positivas a este tópico apresentaram hipertrofia das tonsilas palatinas, a amigdalite de repetição merece atenção especial por parte da área de saúde, visto que pode ser um indício deste quadro, com risco de acontecer juntamente com a hipertrofia adenoidiana ${ }^{10,16,25}$.

Dos adolescentes que referiram amigdalite de repetição, $60 \%$ apresentaram o diagnóstico de
TDAH do Tipo II hiperativo e $54,5 \%$, do Tipo I predominantemente desatento. São escassas as pesquisas sobre a relação entre a amigdalite e o TDAH, porém, em um estudo recente observou-se que a obstrução das vias aéreas superiores pode levar a efeitos adversos no comportamento ${ }^{26}$, podendo haver melhora considerável nos escores de testes comportamentais quando aplicados antes e após o tratamento adequado ${ }^{27}$.

Mesmo não especificando a etiologia em relação à alteração do modo respiratório, um estudo recente observou que o tratamento da RPO em sua amostra de TDAH interferiu diretamente na diminuição dos sintomas de hiperatividade e desatenção ${ }^{23}$.

Em relação aos sintomas da RPO e o gênero dos adolescentes, houve diferença significante no sintoma de sialorreia durante o sono, predominando no gênero masculino ${ }^{10,16}$.

$\mathrm{Na}$ análise da relação entre os sintomas da RPO e faixa etária, houve diferença significativa na respiração oral noturna, respiração oral diurna ${ }^{21}$ e sialorreia ${ }^{10}$, assim como em relação à faixa etária e aos sinais de lábios não funcionais ${ }^{16}$ e língua não funcional ${ }^{22}$. Outros estudos apresentam resultados que discordam dos registrados nesta pesquisa, no que se refere à relação entre sintomas e sinais da RPO e o gênero ${ }^{11} \mathrm{e}$ este com a faixa etária ${ }^{20}$.

Esta pesquisa inclui adolescentes em sua amostra, abrindo um campo novo para futuros estudos, tendo em vista que a maioria dos trabalhos publicados sobre os sintomas e sinais característicos da respiração predominantemente oral foi desenvolvida em crianças.

As queixas de prejuízos escolares foram consideradas, neste estudo, como indicadores do desfecho do desempenho na escola, os quais podem ser observados em número de repetências, suspensões, notas baixas, expulsões, inclusões em classes especiais, entre outros parâmetros ${ }^{1}$.

Ao ser analisada a relação entre os tipos de TDAH e prejuízos escolares neste estudo, constatou-se diferença estatisticamente significante no que concerne a expulsões, suspensões e advertências ${ }^{6-8}$.

Estes dados estão em consonância com a literatura, visto que o prejuízo escolar no TDAH pode estar diretamente relacionado ao comportamento em sala de aula ${ }^{1,6}$.

Apesar dos três tipos de TDAH estarem associados ao prejuízo escolar, há particularidades. O Tipo II - hiperativo apresenta dificuldade de sustentação da atenção, com maior vulnerabilidade à distração, o que ocasiona mais problemas de disciplina do que de desempenho ${ }^{28}$. Na pesquisa, esta afirmação foi evidenciada, posto que, no Tipo II, houve uma maior ocorrência de expulsões, suspensões e 
advertências, aspectos relacionados ao mau comportamento em sala de aula.

Embora um elevado percentual da amostra apresentasse queixas respiratórias, menos da metade realizou algum tratamento, fato este que pode ser justificado em parte devido ao difícil acesso à saúde pública ${ }^{21}$. Por outro lado, apesar dos sintomas da RPO serem persistentes e incômodos, não são estes que fazem os pais procurarem ajuda, mas as consequências decorrentes do quadro, como o prejuízo escolar ${ }^{19}$.

As dificuldades escolares fazem parte do quadro clínico do respirador oral ${ }^{5,19}$ e apesar do tipo de obstrução proporcionar diferentes sintomas, seja por obstruções provocadas pelas tonsilas ${ }^{10}$, ou desconforto devido à rinite alérgica ${ }^{17}$, a perda na qualidade do sono vai influenciar diretamente no comportamento ${ }^{29}$ e a vida escolar no tocante à disciplina e ao rendimento na aprendizagem ${ }^{5,19}$.

A qualidade de vida em pacientes que apresentam respiração oral é bastante afetada. Não apenas a respiração oral, como também, outras doenças respiratórias como a asma e a rinite alérgica trazem prejuízos funcionais, físicos e comportamentais ${ }^{17}$, o que consequentemente vai interferir na vida escolar.

Os sintomas de respiração oral noturna bem como os sinais de língua e lábios alterados podem ocasionar a abertura constante da boca e sialorreia no travesseiro, prejudicando o sono ${ }^{9}$. Estas alterações em geral não são percebidas e, em muitos casos, o ronco surge como o único sintoma referido pelos pais, devido ao incômodo decorrente de sua manifestação sonora. Apesar de não ser observada, na análise dos dados desta pesquisa, diferença significante em relação ao ronco, este foi referido por mais da metade dos adolescentes, podendo ser indício dos DRS, devendo ser investigado em pacientes com RPO ${ }^{5,19,29}$.

Estudos sugerem a relação entre alterações do sono e mudança de comportamento em portadores de TDAH, podendo ser uma das causas para o surgimento de alterações comportamentais ou mesmo para intensificá-las ${ }^{30}$, visto que existe uma associação entre os distúrbios do sono com a hiperatividade, o déficit de atenção e a agressividade ${ }^{26}$.

Por outro lado, em pacientes portadores de comportamento de desatenção e hiperatividade, as doenças como asma, alergia respiratória, infecções respiratórias frequentes e hipertrofia adenoidiana devem ser consideradas, visto que podem levar a efeitos adversos no comportamento ${ }^{26}$.

Como se pode observar existe uma íntima relação entre a RPO, as alterações do sono e o desempenho escolar. Por outro lado há estudos que sugerem uma correlação entre as alterações no sono, o TDAH e a influência destes na vida escolar ${ }^{30,31}$.
Apesar de serem escassas as pesquisas que abordam estes temas em um mesmo estudo, recentemente foi encontrada a associação entre TDAH, baixo rendimento escolar e presença de respiração oronasal devido a presença de comorbidade com o transtorno de aprendizagem ${ }^{11}$.

Em outro estudo o tratamento ortopédico funcional para a respiração oral juntamente com a terapia fonoaudiológica mostrou-se efetivo para melhora dos sintomas de TDAH nos paciente em tratamento com metilfenidato ${ }^{23}$.

Algumas variáveis não foram totalmente elucidadas nesta pesquisa, sendo imprescindíveis abordálas em estudos futuros.

Entretanto, baseado nas informações dos resultados desta pesquisa, é provável haver uma íntima relação entre a RPO e os DRS e entre estes e o TDAH, repercutindo diretamente no desempenho escolar.

Convém ressaltar que o tamanho da amostra estudada não permite tratamento estatístico que forneça dados epidemiológicos significantes.

Ao longo desta pesquisa foi mostrada uma elevada ocorrência de sintomas e sinais da RPO na amostra composta por adolescentes que apresentavam o diagnóstico do TDAH e a queixa de prejuízo escolar. Com base nestes achados, julga-se importante a avaliação do modo respiratório em todos os pacientes que apresentem o TDAH e prejuízo escolar, tendo em vista que as dificuldades escolares podem estar associadas à história pregressa de respiração oral na infância e aos DRS quando encontrados nestes dois quadros ${ }^{31}$.

\section{CONCLUSÃO}

Com base nos resultados desta pesquisa, foi possível concluir em relação à amostra estudada que:

- Os adolescentes com TDAH e queixa de prejuízo escolar apresentam uma alta frequência de características da RPO

- As características da RPO encontradas em mais da metade da amostra foram ronco, respiração oral noturna, rinite, sialorreia no travesseiro, olheiras e lábios ressecados.

- Em relação aos sintomas da RPO, comprovouse a associação significante entre amigdalite e os tipos de TDAH; entre sialorreia no travesseiro e o gênero, e entre respiração oral diurna, respiração oral noturna, sialorreia no travesseiro e faixa etária dos adolescentes.

- Em relação aos sinais da RPO, comprovou-se a associação entre lábios e língua não funcionais e a faixa etária. 


\begin{abstract}
Purpose: to characterize symptoms and signs of Mouth Breathing in children with Attention Deficit Hyperactivity Disorder and with complaints about school underachievement. The specific objectives were to relate the found characteristics to the Attention Deficit Hyperactivity Disorder types, gender and age of this population. Methods: data were collected at ADOLESCENTRO; a public institution of the State Secretary of Health of the Federal District - Brazil. Nocturne and daytime symptoms that appeared in the age ranging between two and twelve-years-old were reported by the parents and were analyzed, as well as some signs cited on literature as being common characteristics of Mouth Breathing. Results: a high frequency of Mouth Breathing characteristics were found to be associated with snoring, nocturne mouth breathing, rhinitis, sialorrhoea, dark undereye circles and dry lips. Related to the symptoms, an association was found among tonsillitis and Attention Deficit Hyperactivity Disorder types; sialorrhoea on the pillow with masculine gender and daytime mouth breathing; nocturne mouth breathing, sialorrhoea on the pillow with age. An association was found between non-functional lips with age and non-functional tongue with age. Conclusion: the possibility of simultaneous existence of Mouth Breathing on children with Attention Deficit Hyperactivity Disorder and complaints about school underachievement requires a deeper diagnostic investigation.
\end{abstract}

KEYWORDS: Attention Deficit Disorders With Hyperactivity; Mouth Breathing; Sleep; Respiration Disorders; Underachievement; Adolescent

\section{REFERÊNCIAS}

1. Pastura GMC, Mattos $P$, Araújo APQC. Desempenho escolar e transtorno do déficit de atenção e hiperatividade. Rev Psiquiatr Clin. 2005; 32(6):324-9.

2. Mattos P. Transtorno do déficit de atenção e hiperatividade. Rev Pedriatr Moder. 2005; 41(2):55-63.

3. Silva RAS, Souza IAP. Aspectos linguísticos e sociais relacionados ao transtorno do déficit de atenção/hiperatividade. Rev. CEFAC. 2005; 7(3):295-9.

4. Schmitz M, Polanczyk G, Rohde LAP. TDAH: remissão na adolescência e preditores de persistência em adultos. J Bras Psiquiatr. 2007; 56(Supl1):25-9.

5. Marchesan I. Q. Avaliação e terapia dos problemas da respiração. In: Marchesan IQ. Fundamentos em fonoaudiologia: aspectos clínicos da motricidade oral. Rio de Janeiro: Guanabara Koogan; 1998. p. 23-36.

6. Barkley RA, Fischer M, Edelbrock CS, Smallish $\mathrm{L}$. The adolescent outcome of hyperactive children diagnosed by research criteria: I. an 8-year prospective follow-up study. J Am Acad Chid Adolesc Psychiatr. 1990; 29(4):546-57.

7. Faraone SV, Biederman J, Lehman BK, Spencer T, Norman D, Seidman LJ, et al. Intellectual performance and school failure in children with attention deficit hyperactivity disorder and in their siblings. J Abnorm Psychol. 1993; 102(4):616-23.
8. Rohde LA, Halpern R. Transtorno de deficit de atenção/hiperatividade: atualização. J Pediatr. 2004; 80(Supl2):61-70.

9. Amaral AK, Carvalho MM, Justino HS. Respiração oral e suas relações com ronco noturno e a síndrome da apnéia obstrutiva do sono. J Bras Fonoaudiol. 2004; 5(18):60-2.

10. Di Francesco RC, Passerotii G, Paulucci B, Miniti A. Respiração oral na criança: repercussões diferentes de acordo com o diagnóstico. Rev Bras Otorrinolaringol. 2004; 70(5):665-70.

11. Vera CFD, Conde GLS, Wajnsztejn R, Nemr K. Transtornos de aprendizagem em presença de respiração oral em indivíduos com diagnóstico de transtorno de déficit de atenção/ hipertividade (TDAH). Rev. CEFAC. 2006; 8(4):441-55. dx.doi. org/10.1590/S1516-18462006000400005

12. Possa MA, Spanemberg L, Guardiola A. Comorbidades do transtorno de déficit de atenção e hiperatividade em crianças escolares. Arq Neuropsiquiatr. 2005; 63(2B):479-83.

13. Poeta LS, Neto Rosa FR. Estudo epidemiológico dos sintomas do transtorno do déficit de atenção/ hiperatividade e transtornos de comportamento em escolares da rede pública de Florianópolis usando a EDAH. Rev Bras Psiquiatr. 2004; 26(3):150-5.

14. Cardoso LF, Sabbag S, Beltrame TS. Prevalência de transtorno de déficit de atenção/hiperatividade em relação ao gênero de escolares. Rev Bras Cineantropom Desemp Hum. 2007; 9(1):50-4. 
15. Malerbi FF, Scheuer Cl. Memória implícita em crianças com TDA/H. Rev Soc Bras Fonoaudiol. 2005; 10(3):133-8.

16. Branco A, Ferrari GF, Weber SAT. Alterações orofaciais em doenças alérgicas de vias aéreas: [revisão]. Rev Paul Pediatr. 2007; 25(3):266-70.

17. Campanha SMA, Freire LMS, Fontes MJF. O impacto da asma, da rinite alérgica

e da respiração oral na qualidade de vida de crianças e adolescentes. Rev. CEFAC. 2008; 10(4):513-9. dx.doi.org/10.1590/S1516-18462008000400011

18. Borges WG, Burns DAR, Felizola MLBM, Oliveira BA, Hamu CS, Freitas VC. Prevalência de rinite alérgica em adolescentes do Distrito Federal: comparação entre as fases I e I| do ISAAC. J Pediatr. 2008; 82(2):137-43.

19. Chedid KAK, Di Francesco RC, Junqueira PAS. A influência da respiração oral no processo de aprendizagem da leitura e escrita em crianças préescolares. Rev Psicoped. 2004; 21(65):157-63.

20. Menezes VA, Leal RB, Pessoa RS, Pontes RMES. Prevalência e fatores associados à respiração oral em escolares participantes do projeto Santo Amaro- Recife, 2005. Rev Bras Otorrinolaringol. 2006; 72(3):394-9.

21. Araújo AS, Moura JR, Camargo LA. Principais sintomas otorrinolaringológicos em escolares. Arq ORL. 2004; 8 (1):262-6.

22. Rodrigues HOSN, Faria SR, Paula FSG, Motta AR. Ocorrência de respiração oral e alterações miofuncionais orofaciais em sujeitos em tratamento ortodôntico. Rev. CEFAC. 2005; 7(3):356-62.

23. Costa CMF. Influência do tratamento da respiração oral na sintomatologia de crianças com transtorno do déficit de atenção/hiperatividade. [dissertação]. São Paulo: Universidade de São Paulo; 2007.
24. Melo GMF, Cunha DA, Silva HJ. Avaliação da aeração nasal pré e pós a realização de manobras de massagem e limpeza nasal. Rev. CEFAC. 2007; 9(3):375-82. dx.doi.org/10.1590/ S1516-18462007000300011

25. Santos RS, Cipolotti R, D’Ávila JS, Gurgel RQ. Escolares submetidos a videonasofaringoscopia na escola: achados e aceitação. J Pediatr. 2005; 81(6):443-6.

26. Gottlieb DJ, Vezina RM, Chase C, Lesko SM, Heeren TC, Weese-Mayer DE, et al. Symptoms of sleep-disordered breathing in 5-year-old children are associated with sleepiness and problem behaviors. Pediatrics. 2003; 112(4):870-7.

27. Goldstein NA, Scurelati N, Walsleben JA, Bhatia $\mathrm{N}$, Friedman D, Rapoport DM. Clinical diagnosis of pediatric obstructive sleep apnea validated by polissomnography. Otolaryngol Head Neck Surg. 1994; 111(5):611-7.

28. Souza I, Serra MA, Mattos P, Franco VA. Comorbidade em crianças e adolescentes com transtorno do déficit de atenção: resultados preliminares. Arq Neuropsiquiatr. 2001; 59(2B):401-6.

29. O'Brien LM, Mervis CB, Holbrook CR, Bruner JL, Klaus CJ, Rutherford J, et al. Neurobehavioral implications of habitual snoring in children. Pediatrics. 2004; 114(1):44-9.

30. Neves SNH, Reimão R. Sleep disturbances in 50 children with attention-deficit hyperactivity disorder. Arq Neuropsiquiatr. 2007; 65(2A):228-33. 31. Gozal D, Pope Junior DW. Snoring during early childhood and academic performance at ages thirteen to fourteen years. Pediatrics. 2001; 107(6):1394-9.

RECEBIDO EM: 27/02/2009

ACEITO EM: 15/09/2009

Endereço para correspondência:

Tatiana Leonel da Silva Costa

SQN, 411 BI H ap. 206 Asa Norte

Brasília - DF

CEP: 70866-080

E-mail: tatianafono@globo.com 